\title{
Convergence Analysis of Semi-Implicit Euler Methods for Solving Stochastic Age-Dependent Capital System with Variable Delays and Random Jump Magnitudes
}

\author{
Qinghui $\mathrm{Du}^{1}$ and Chaoli Wang ${ }^{2}$ \\ ${ }^{1}$ Business School, University of Shanghai for Science and Technology, Shanghai 200093, China \\ ${ }^{2}$ Department of Control Science and Engineering, University of Shanghai for Science and Technology, Shanghai 200093, China \\ Correspondence should be addressed to Chaoli Wang; clclwang@126.com
}

Received 29 July 2014; Revised 14 October 2014; Accepted 14 October 2014; Published 13 November 2014

Academic Editor: Hari M. Srivastava

Copyright (C) 2014 Q. Du and C. Wang. This is an open access article distributed under the Creative Commons Attribution License, which permits unrestricted use, distribution, and reproduction in any medium, provided the original work is properly cited.

We consider semi-implicit Euler methods for stochastic age-dependent capital system with variable delays and random jump magnitudes, and investigate the convergence of the numerical approximation. It is proved that the numerical approximate solutions converge to the analytical solutions in the mean-square sense under given conditions.

\section{Introduction}

Stochastic differential equations have been widely used to model the phenomena arising in many branches of science and industry fields such as biology, economic, finance, and ecology [1-4]. Recently, the numerical construction of stochastic age-dependent capital system has received a great deal of attention [5-9]. In [8], exponential stability of numerical solutions for a class stochastic age-dependent capital system with Poisson jumps was studied by Zhang et al. in the case of deterministic magnitude. Zhang and Rathinasamy [9] studied convergence of numerical solutions for a class of stochastic age-dependent capital system with random jump magnitudes, which extended the analysis in [8] to the case where the jump magnitudes are random. However, in many real problems, the capital systems can be modeled by stochastic dynamical systems whose evolutions depend not only on the current states, but also on their historical states. So we need a stochastic delay model including an extra term, which is called time delay, to simulate them. In this paper, we consider stochastic age-dependent capital system with variable delays and random jump magnitudes:

$d K(a, t)$

$$
=-\mu(a, t) K(a, t) d t+f(t, K(a, t), K(a, t-\tau(t))) d t
$$

$$
\begin{gathered}
+g(t, K(a, t), K(a, t-\tau(t))) d W(t) \\
+h\left(t, K(a, t), K(a, t-\tau(t)), \gamma_{N(t)+1}\right) d N(t), \\
\text { in } D, \\
K(a, t)=\psi(a, t), \quad \text { in }[-r, 0], \quad \text { in } t \in[0, T], \\
K(0, t)=\varphi(t)=\alpha(t) B(t) F\left(L(t), \int_{0}^{A} K(a, t) d a\right), \\
K(a, 0)=K_{0}(a), \quad \text { in } a \in[0, A], \\
N(t)=\int_{0}^{A} K(a, t) d a, \quad \text { in } t \in[0, T],
\end{gathered}
$$

where $d K(a, t)=(\partial K(a, t) / \partial t+\partial K(a, t) / \partial a) d t, \varphi(0)=K_{0}(0)$, and $D=[0, A] \times[0, T]$. The stock of capital goods of age $a$ at time $t$ is denoted by $K(a, t)$. This makes that total output produced at time $t$ defined as $N(t)$; also $a$ is the age of the capital, the investment $\varphi(t)$ in the new capital. $f(t, K(a, t)$, 
$K(a, t-\tau(t)))+g(t, K(a, t), K(a, t-\tau(t))) d W(t)+h(t, K(a, t)$, $\left.K(a, t-\tau(t)), \gamma_{N(t)+1}\right) d N(t)$ denote effects of the external environment for capital system, such as innovations in techniques, natural disasters, introduction of new products, and changes in laws and government policies, and so on. $\tau(t)$ is a variable delay, and the $f$ is the appreciation (when $f \geq 0$ ) or depreciation (when $f \leq 0$ ) of the production capacity, and $g$ represents the volatility of the capital stock. $W(t)$ is a standard Wiener process. $N(t)$ is a Poisson process with mean $\lambda t$ and $\gamma_{i}, i=1,2, \ldots$, are independent, identically distributed random variables representing magnitudes for each jump. We assume that for some $p \geq 2$ there is a constant $C$ such that $E\left[\left|\gamma_{i}\right|^{p}\right] \leq C$; that is, the $p$ th moment of the jump magnitude is bounded. The maximum physical lifetime of capital $A$, the planning interval of calendar time $[0, T]$, the depreciation rate $\mu(a, t)$ of capital, and the capital density $K_{0}(a)$ (the initial distribution of capital over age) are given. The $\alpha(t)$ denotes the accumulative rate of capital at the moment of $t, 0<$ $\alpha(t)<1$, and $B(t)$ is the technical progress at the moment of $t$. Each sector of all the firms has an identical neoclassical technology and produces output using labor and capital. The production function $F\left(L(t), \int_{0}^{A} K(a, t) d a\right)$ is neoclassical, where $\int_{0}^{A} K(a, t) d a$ is the total sum of capital at time $t$ and $L(t)$ is the labor force.

In general most equations of stochastic age-dependent capital system with variable delays and random jump magnitudes do not have explicit solution. Thus, numerical approximation schemes are invaluable tools for exploring its properties. There is a significant amount of literature that has been published concerning approximate schemes for stochastic differential equations with jumps [9-11] or stochastic differential delay equations [12-14]. In [15], Chalmers and Higham gave the semi-implicit Euler approximate solutions and proved the convergence of semi-implicit Euler methods under the Lipschitz condition. However, in many situations, the coefficients $f, g$, and $h$ are only locally Lipschitz continuous. It is therefore useful to establish the strong convergence of the semi-implicit Euler method under the local Lipschitz condition. In this paper, we relax the global Lipschitz condition on the coefficients which was imposed in [15] and prove that the semi-implicit Euler approximate solutions converge to the exact solutions of (1) in the meansquare sense under the local Lipschitz condition.

In Section 2, we will collect some notation and hypotheses concerning (1), and the semi-implicit Euler method is used to produce numerical solutions. In Section 3, we give the useful lemmas which are essential to prove our main results, that is, Theorem 17.

\section{Preliminaries and the Semi-Implicit Euler Approximation}

Throughout this paper, it will be denoted by $L^{2}([0, A])$ the space of functions that are square-integrable over the domain $[0, A]$. Let

$$
V=H^{1}([0, A])
$$

$$
\begin{aligned}
& \equiv\left\{\varphi \mid \varphi \in L^{2}([0, A]), \frac{\partial \varphi}{\partial a} \in L^{2}([0, A]),\right. \\
& \text { where } \left.\frac{\partial \varphi}{\partial a} \text { are generalized partial derivatives }\right\} .
\end{aligned}
$$

$V$ is the Sobolev space. $H=L^{2}([0, A])$ such that $V \rightarrow$ $H \equiv H^{\prime} \rightarrow V^{\prime} . V^{\prime}=H^{-1}([0, A])$ is the dual space of $V$. We denote by $\|\cdot\|$ and $|\cdot|$ the norms in $V$ and $V^{\prime}$, respectively; by $(\cdot, \cdot)$ the scalar product in $H .\langle\cdot, \cdot\rangle$, the duality product between $V$ and $V^{\prime}$, is defined by

$$
\langle\cdot, \cdot\rangle=\int_{0}^{A} u \cdot v d a, \quad u \in V, v \in V^{\prime} .
$$

Let $W(t)$ be a Wiener process defined on complete probability space $(\Omega, F, P)$ with covariance operator $G$ and taking its values in the separable Hilbert space $S$ :

$$
W(t)=\sum_{i=1}^{\infty} \beta_{i}(t) e_{i}
$$

where $\left\{e_{i}\right\}_{i \geq 0}$ is an orthonormal set of eigenvectors of $G, \beta_{i}(t)$ that are mutually independent real Wiener processes with incremental covariance $\lambda_{i}>0, G e_{i}=\lambda_{i} e_{i}$, and $\operatorname{tr} G=\sum_{i=1}^{\infty} \lambda_{i}$ (tr denotes the trace of an operator). For an operator $A \in$ $\phi(S, H)$ is the space of all bounded linear operators from $S$ into $H$; it is denoted by $\|A\|_{2}$; its denotes the Hilbert-Schmidt norm; that is,

$$
\|A\|_{2}^{2}=\operatorname{tr}\left(A G A^{T}\right)
$$

Let $C=C([0, T] ; H)$ be the space of all continuous function from $[0, T]$ into $H$ with sup-norm $\|\varphi\|_{c}=\sup _{0 \leq s \leq T}|\varphi(s)|$, $L_{V}^{p}=L^{p}([0, T] ; V)$, and $L_{H}^{p}=L^{p}([0, T] ; H)$.

Let $(\Omega, F, P)$ be a complete probability space with a filtration $\left\{F_{t}\right\}_{t \geq 0}$ satisfying the usual conditions (i.e., it is increasing and right continuous with a left-hand side limit, and $F_{0}$ contains all $P$-null sets). Let $N(t)$ be a scalar Poisson process with intensity $\lambda$ which is independent from Wiener process $W(t)$. Denote by $D_{F_{0}}^{p}([-r, 0] ; H)$ the family of all bounded, $F_{0}$-measurable, and $D([-r, 0] ; H)$-valued random variables. Let $p>0, t \geq 0$, and $D_{F_{0}}^{p}([-r, 0] ; H)$ denote the family of all $F_{0}$-measurable, $D([-r, 0] ; H)$-valued variables $\psi$ which satisfies sup ${ }_{-r \leq s \leq 0} E|\psi(s)|^{p}<\infty$. In this paper, $f(t, \cdot, \cdot)$, $g(t, \cdot, \cdot)$, and $h(t, \cdot, \cdot)$ are family of nonlinear operators, $F_{t^{-}}$ measurable almost surely in $t$.

The integral version of (1) is given by

$$
\begin{aligned}
K_{t}= & K_{0}-\int_{0}^{t} \frac{\partial K_{s}}{\partial a} d s-\int_{0}^{t} \mu(a, s) K_{s} d s \\
& +\int_{0}^{t} f\left(t, K_{s}, K_{s-\tau(s)}\right) d s+\int_{0}^{t} g\left(t, K_{s}, K_{s-\tau(s)}\right) d W(s) \\
& +\int_{0}^{t} h\left(t, K_{s}, K_{s-\tau(s)}, \gamma(s)\right) d N(s) .
\end{aligned}
$$


For system (6), the discrete semi-implicit Euler approximation on $t \in\{0, h, 2 h, \ldots\}$ is given by the iterative scheme

$$
\begin{aligned}
& Q_{n+1} \\
& =Q_{n}-\frac{\partial Q_{n+1}}{\partial a} h+(1-\theta) \\
& \quad \times\left[-\mu\left(t_{n}, a\right) Q_{n}+f\left(Q_{n}, Q_{[(n h-\tau(n h)) / h]}\right)\right] h \\
& \quad+\theta\left[-\mu\left(t_{n}, a\right) Q_{n+1}+f\left(Q_{n+1}, Q_{[((n+1) h-\tau((n+1) h)) / h]}\right)\right] h \\
& \quad+g\left(Q_{n}, Q_{[(n h-\tau(n h)) / h]}\right) \Delta W_{n} \\
& \quad+h\left(Q_{n}, Q_{[(n h-\tau(n h)) / h]}, \gamma_{N\left(t_{n}\right)+1}\right) \Delta N_{n},
\end{aligned}
$$

with initial value $Q_{0}=K(0, a)=K_{0} ; 0 \leq \theta \leq 1$; and $[u]$ represents the integer part of $u$. Here the time increment is $h=T / m$, for some sufficiently large integer $m$ such that $h \ll 1$ and $Q_{n} \approx K\left(a, t_{n}\right)$, for $h=t_{n+1}-t_{n}$, and $\Delta W_{n}=W\left(t_{n+1}\right)-$ $W\left(t_{n}\right)$ and $\Delta N_{n}=N\left(t_{n+1}\right)-N\left(t_{n}\right), n=0,1,2, \ldots, m$, are the Wiener and Poisson increments, respectively.

Define the step functions:

$$
\begin{gathered}
\bar{Y}_{t}=\bar{Y}(t, a)=\sum_{n=0}^{m-1} Q_{n} I_{[n h,(n+1) h)}(t), \\
\widehat{Y}_{t}=\widehat{Y}(t, a)=\sum_{n=0}^{m-1} Q_{[(n h-\tau(n h)) / h]} I_{[n h,(n+1) h)}(t), \\
\bar{Z}_{t}=\bar{Z}(t, a)=\sum_{n=0}^{m-1} Q_{n+1} I_{[n h,(n+1) h)}(t), \\
\widehat{Z}_{t}=\widehat{Z}(t, a)=\sum_{n=0}^{m-1} Q_{[((n+1) h-\tau((n+1) h)) / h]} I_{[n h,(n+1) h)}(t), \\
\bar{\gamma}(t)=\sum_{n=0}^{m-1} \gamma_{N\left(t_{n}\right)+1} I_{[n h,(n+1) h)}(t),
\end{gathered}
$$

where $I_{A}$ is the indicator function for set $A$. Then we define the continuous semi-implicit Euler approximation:

$$
\begin{aligned}
Q_{t}= & K_{0}-\int_{0}^{t} \frac{\partial Q_{s}}{\partial a} d s \\
& +\int_{0}^{t}(1-\theta)\left[-\mu(s, a) \bar{Y}_{s}+f\left(\bar{Y}_{s}, \widehat{Y}_{s}\right)\right] d s \\
& +\int_{0}^{t} \theta\left[-\mu(s, a) \bar{Z}_{s}+f\left(\bar{Z}_{s}, \widehat{Z}_{s}\right)\right] d s \\
& +\int_{0}^{t} g\left(\bar{Y}_{s}, \widehat{Y}_{s}\right) d W(s) \\
& +\int_{0}^{t} h\left(\bar{Y}_{s}, \widehat{Y}_{s}, \bar{\gamma}(s)\right) d N(s)
\end{aligned}
$$

Remark 1. If the parameter $\theta=0$ in (7), then the semiimplicit Euler methods become the Euler methods, which have been studied in $[10,12,14]$.

In order to establish the convergence theorem, we propose the following assumptions.

Assumption 2. The function $\tau:[0, \infty] \rightarrow R$ is the time delay which satisfies

$$
\tau(t) \geq 0, \quad t-\tau(t) \geq-r .
$$

For $t, s \geq 0$, there exists a constant $k<1$ such that

$$
|\tau(t)-\tau(s)| \leq k|t-s|
$$

Assumption 3 (local Lipschitz condition). There exist two positive constants $K_{d}, K$ such that, for all $x_{1}, y_{1}, x_{2}, y_{2}$, $z_{1}, z_{2} \in H$ and $t \in[0, T]$,

$$
\begin{aligned}
& \left|f\left(x_{1}, y_{1}\right)-f\left(x_{2}, y_{2}\right)\right|^{2} \vee\left\|g\left(x_{1}, y_{1}\right)-g\left(x_{2}, y_{2}\right)\right\|_{2}^{2} \\
& \quad \leq K_{d}\left(\left|x_{1}-x_{2}\right|^{2}+\left|y_{1}-y_{2}\right|^{2}\right), \\
& \left|h\left(x_{1}, y_{1}, z_{1}\right)-h\left(x_{2}, y_{2}, z_{2}\right)\right|^{2} \\
& \quad \leq K_{d}\left(\left|x_{1}-x_{2}\right|^{2}+\left|y_{1}-y_{2}\right|^{2}\right)+K\left(\left|z_{1}-z_{2}\right|^{2}\right) .
\end{aligned}
$$

Assumption 4. $\mu(t, a)$ is a nonnegative measurable function in $D$ such that

$$
0 \leq \mu_{0} \leq \mu(t, a) \leq \bar{\alpha}<\infty
$$

and $B(t)$ is a nonnegative continuous function in $[0, T]$ such that $\alpha(t) B(t) \leq \eta ; \eta$ is a nonnegative constant in $[0, T]$.

Assumption 5. The initial function $\psi$ is Holder-continuous with exponent $\gamma$; that is, there exists a positive constant $K^{\prime}$ such that, for $t, s \in[-\tau, 0]$,

$$
E\left(|\psi(t)-\psi(s)|^{2}\right) \leq K^{\prime}|t-s|^{2 \gamma} .
$$

Assumption 6. Consider $f(t, 0)=0, g(t, 0)=0$, and $h(t, 0$, $\left.\gamma_{N(t)+1}\right)=0, t \in[0, T]$.

Assumption 7. Consider $F(L, N) \geq 0,(F(L, 0)=0), \partial F / \partial L>$ 0 , and $0<\partial F / \partial N \leq F_{1}$, where $F_{1}$ is a positive constant.

Remark 8. If the local Lipschitz condition holds, then there exists a positive constant $K_{d}^{\prime}$ such that, for $x, y, z \in R^{n}$,

$$
|f(x, y)|^{2} \vee\|g(x, y)\|_{2}^{2} \vee|h(x, y, z)|^{2} \leq K_{d}^{\prime}
$$

\section{Convergence of the Semi-Implicit Euler Approximate Solution}

In this section, several lemmas which are useful for the following main result are given. 
Lemma 9. Under Assumptions 2-7, there are constants $k \geq 2$ and $C_{1}>0$ such that

$$
E\left[\sup _{0 \leq t \leq T}\left|K_{t}\right|^{k}\right] \leq C_{1}
$$

The proof is similar to that of in [16].

Let $\sigma_{n}=\inf \left\{t \geq 0:\left|K_{t}\right| \geq n\right\}$ and $\rho_{n}=\inf \left\{t \geq 0:\left|Q_{t}\right| \geq\right.$ $n\}$. Define the stopping time $v_{n}=\rho_{n} \wedge \sigma_{n}$.

Lemma 10. Under Assumptions 2-7, there exists a constant $C_{2}>0$ such that

$$
E\left[\sup _{t \in[0, T]}\left|Q_{t \wedge v_{n}}\right|^{2}\right] \leq C_{2} .
$$

Proof. From (9), applying Itô formula to $\left|Q_{t \wedge v_{n}}\right|^{2}$ yields

$$
\begin{aligned}
\left|Q_{t \wedge v_{n}}\right|^{2} & \left|Q_{0}\right|^{2}+2 \int_{0}^{t \wedge v_{n}}\left\langle-\frac{\partial Q_{s}}{\partial a}, Q_{s}\right\rangle d s \\
& -2 \int_{0}^{t \wedge v_{n}}\left(Q_{s}, \mu(s, a)\left[(1-\theta) \bar{Y}_{s}+\theta \bar{Z}_{s}\right] d s\right) \\
& +2 \int_{0}^{t \wedge v_{n}}\left(Q_{s},\left[(1-\theta) f\left(\bar{Y}_{s}, \widehat{Y}_{s}\right)+\theta f\left(\bar{Z}_{s}, \widehat{Z}_{s}\right)\right] d s\right) \\
& +\int_{0}^{t \wedge v_{n}}\left\|g\left(\bar{Y}_{s}, \widehat{Y}_{s}\right)\right\|_{2}^{2} d s+2 \int_{0}^{t \wedge v_{n}}\left(Q_{s}, g\left(\bar{Y}_{s}, \widehat{Y}_{s}\right) d W_{s}\right) \\
& +2 \int_{0}^{t \wedge v_{n}}\left(Q_{s}, h\left(\bar{Y}_{s}, \widehat{Y}_{s}, \bar{\gamma}(s)\right)\right) d N(s) \\
& +\int_{0}^{t \wedge v_{n}}\left|h\left(\bar{Y}_{s}, \widehat{Y}_{s}, \bar{\gamma}(s)\right)\right|^{2} d N(s) \\
\leq & \left|Q_{0}\right|^{2}+2 \int_{0}^{t \wedge v_{n}}\left\langle-\frac{\partial Q_{s}}{\partial a}, Q_{s}\right\rangle d s \\
& +2 \int_{0}^{t \wedge v_{n}}\left(Q_{s}, \mu(s, a)\left[(1-\theta) \bar{Y}_{s}+\theta \bar{Z}_{s}\right] d s\right) \\
& +2 \lambda \int_{0}^{t \wedge v_{n}}\left(Q_{s}, h\left(\bar{Y}_{s}, \widehat{Y}_{s}, \bar{\gamma}(s)\right) d s\right) \\
& +2 \int_{0}^{t \wedge v_{n}}\left(Q_{s},\left[(1-\theta) f\left(\bar{Y}_{s}, \widehat{Y}_{s}\right)+\theta f\left(\bar{Z}_{s}, \widehat{Z}_{s}\right)\right] d s\right) \\
& +\int_{0}^{t \wedge v_{n}}\left\|g\left(\bar{Y}_{s}, \widehat{Y}_{s}\right)\right\|_{2}^{2} d s+2 \int_{0}^{t \wedge v_{n}}\left(Q_{s}, g\left(\widehat{Y}_{s}, \widehat{Y}_{s}, \bar{Y}_{s}\right) d W_{s}\right) \\
& +2(s))\left.\right|^{2} d \bar{N}_{s}(s), \\
& \left.+Q_{s}, h\left(\bar{Y}_{s}, \widehat{Y}_{s}, \bar{\gamma}(s)\right) d \bar{N}_{N}(s)\right)
\end{aligned}
$$

where $N(t)=\bar{N}(t)-\lambda t$ is a compensated Poisson process. Note

$$
\begin{aligned}
& \left\langle-\frac{\partial Q_{s}}{\partial a}, Q_{s}\right\rangle \\
& =-\int_{0}^{A} Q_{s} \cdot \frac{\partial Q_{s}}{\partial a} d a \\
& =\frac{1}{2} \alpha^{2}(s) B^{2}(s)\left[F\left(L(s), \int_{0}^{A} Q_{s} d a\right)-F(L(s), 0)\right]^{2} \\
& \leq \frac{1}{2} \eta^{2}\left(\left.\frac{\partial F(L, N)}{\partial N}\right|_{y}\right)^{2}\left(\int_{0}^{A} Q_{s} d a\right) \\
& \leq \frac{1}{2} A F_{1}^{2} \eta^{2}\left|Q_{s}\right|^{2},
\end{aligned}
$$

where $y \in\left(0, \int_{0}^{A} Q_{s} d a\right)$.

Therefore, by Assumption 4, (15), and a variant of Cauchy-Schwarz inequality for any $t \in[0, T]$, along with (18), we get that

$$
\begin{aligned}
& \left|Q_{t \wedge v_{n}}\right|^{2} \\
& \leq\left|Q_{0}\right|^{2}+\left(A F_{1}^{2} \eta^{2}+1+\bar{\alpha}+\lambda\right) \\
& \quad \times \int_{0}^{t \wedge v_{n}}\left|Q_{s}\right|^{2} d s+2 \bar{\alpha} \int_{0}^{t \wedge v_{n}}\left[\left|\bar{Y}_{s}\right|^{2}+\left|\bar{Z}_{s}\right|^{2}\right] d s \\
& \quad+(5+\lambda) K_{d}^{\prime} T+2 \int_{0}^{t \wedge v_{n}}\left(Q_{s}, g\left(\bar{Y}_{s}, \widehat{Y}_{s}\right) d W_{s}\right) \\
& \quad+2 \int_{0}^{t \wedge v_{n}}\left(Q_{s}, h\left(\bar{Y}_{s}, \widehat{Y}_{s}, \bar{\gamma}(s)\right) d \bar{N}(s)\right) \\
& \quad+\lambda \int_{0}^{t \wedge v_{n}}\left|h\left(\bar{Y}_{s}, \widehat{Y}_{s}, \bar{\gamma}(s)\right)\right|^{2} d s \\
& \quad+\int_{0}^{t \wedge v_{n}}\left|h\left(\bar{Y}_{s}, \widehat{Y}_{s}, \bar{\gamma}(s)\right)\right|^{2} d \bar{N}(s) .
\end{aligned}
$$

Let $k_{1}=A F_{1}^{2} \eta^{2}+1+\bar{\alpha}+\lambda, k_{2}=2 \bar{\alpha}$; we have

$$
\begin{aligned}
E\left[\sup _{s \in[0, t]}\left|Q_{s}\right|^{2}\right] \\
\leq E\left|Q_{0}\right|^{2}+\left(k_{1}+2 k_{2}\right) \\
\quad \times \int_{0}^{t \wedge v_{n}} E\left[\sup _{s \in[0, t]}\left|Q_{s}\right|^{2}\right] d s+(5+\lambda) K_{d}^{\prime} T \\
\quad+2 E\left[\sup _{s \in[0, t]} \int_{0}^{t \wedge v_{n}}\left(Q_{u}, g\left(\bar{Y}_{u}, \widehat{Y}_{u}\right) d W(u)\right)\right]
\end{aligned}
$$




$$
\begin{aligned}
& +2 E\left[\sup _{s \in[0, t]} \int_{0}^{t \wedge v_{n}}\left(Q_{u}, h\left(\bar{Y}_{u}, \widehat{Y}_{u}, \bar{\gamma}(u)\right) d \bar{N}(u)\right)\right] \\
& +E\left[\sup _{s \in[0, t]} \int_{0}^{t \wedge v_{n}}\left|h\left(\bar{Y}_{u}, \widehat{Y}_{u}, \bar{\gamma}(u)\right)\right|^{2} d \bar{N}(u)\right] .
\end{aligned}
$$

By Burkholder-Davis-Gundy inequality, we have

$$
\begin{aligned}
& E\left[\sup _{s \in[0, t]} \int_{0}^{t \wedge v_{n}}\left(Q_{u}, g\left(\bar{Y}_{u}, \widehat{Y}_{u}\right) d W(u)\right)\right] \\
& \quad \leq \frac{1}{6} E\left[\sup _{s \in[0, t]}\left|Q_{s}\right|^{2}\right]+k_{3} K_{d}^{\prime} T, \\
& E\left[\sup _{s \in[0, t]} \int_{0}^{t \wedge v_{n}}\left(Q_{u}, h\left(\bar{Y}_{u}, \widehat{Y}_{u}, \bar{\gamma}(u)\right) d \bar{N}(u)\right)\right] \\
& E\left[\sup _{s \in[0, t]} \int_{0}^{t \wedge v_{n}}\left|h\left(\bar{Y}_{u}, \widehat{Y}_{u}, \bar{\gamma}(u)\right)\right|^{2} d \bar{N}(u)\right] \leq k_{5} K_{d}^{\prime} T,
\end{aligned}
$$

where $k_{3}, k_{4}$, and $k_{5}$ are positive constants. Substituting (22) into (21) yields, again for a possibly different $k_{6}$ and $k_{7}$,

$$
\begin{aligned}
& E\left[\sup _{s \in[0, t]}\left|Q_{s}\right|^{2}\right] \\
& \quad \leq 3 E\left|Q_{0}\right|^{2}+k_{7} \int_{0}^{t \wedge v_{n}} E\left[\sup _{s \in[0, t]}\left|Q_{s}\right|^{2}\right] d s+k_{6} K_{d}^{\prime} T,
\end{aligned}
$$

for every $t \in[0, T]$. Now, Gronwall lemma obviously implies the required result. The proof is complete.

Lemma 11. Under Assumptions 2-7, there exist constants $k \geq$ 2 and $C_{3}>0$ such that

$$
E\left[\sup _{t \in[0, T]}\left|Q_{t}^{k}\right|\right] \leq C_{3} .
$$

The proof is similar to that of Lemma 9.

Lemma 12. Under Assumption 3 and $E\left|\partial Q_{s} / \partial a\right|^{2} \leq \infty$, for any $t \in[0, T]$,

$$
\begin{aligned}
& E\left[\sup _{t \in[0, T]}\left|Q_{t}-\bar{Y}_{t}\right|^{2}\right] \leq C_{1}(d) h, \\
& E\left[\sup _{t \in[0, T]}\left|Q_{t}-\bar{Z}_{t}\right|^{2}\right] \leq C_{3}(d) h,
\end{aligned}
$$

where $C_{1}(d), C_{3}(d)$ are positive constants dependent only on $\theta$, $\lambda$, and $K_{d}^{\prime}$ and independent of $h$.

Proof. For any $t \in[0, T]$, there exists an integer $n$ such that $t \in[n h,(n+1) h)$; then

$$
\begin{aligned}
Q_{t}-\bar{Y}_{t}= & Q_{t}-Q_{n} \\
= & -\int_{n h}^{t} \frac{\partial Q_{s}}{\partial a} d s-\int_{n h}^{t} \mu(s, a)\left[(1-\theta) \bar{Y}_{s}+\theta \bar{Z}_{s}\right] d s \\
& +\int_{n h}^{t}\left[(1-\theta) f\left(\bar{Y}_{s}, \widehat{Y}_{s}\right)+\theta f\left(\bar{Z}_{s}, \widehat{Z}_{s}\right)\right] d s \\
& +\int_{n h}^{t} g\left(\bar{Y}_{s}, \widehat{Y}_{s}\right) d W(s) \\
& +\int_{n h}^{t} h\left(\bar{Y}_{s}, \widehat{Y}_{s}, \bar{\gamma}(s)\right) d N(s) .
\end{aligned}
$$

Applying the basic inequality $|a+b+c+d+e|^{2} \leq 5|a|^{2}+$ $5|b|^{2}+5|c|^{2}+5|d|^{2}+5|e|^{2}$ to 5 terms on the above right-hand side, we have

$$
\begin{aligned}
& E\left|Q_{t}-\bar{Y}_{t}\right|^{2} \\
& \leq 5 E\left|\int_{k h}^{t} \frac{\partial Q_{s}}{\partial a} d s\right|^{2}+5 E\left|\int_{n h}^{t} \mu(s, a)\left[(1-\theta) \bar{Y}_{s}+\theta \bar{Z}_{s}\right] d s\right|^{2} \\
& \quad+5 E\left|\int_{n h}^{t}\left[(1-\theta) f\left(\bar{Y}_{s}, \widehat{Y}_{s}\right)+\theta f\left(\bar{Z}_{s}, \widehat{Z}_{s}\right)\right] d s\right|^{2} \\
& +5 E\left|\int_{n h}^{t} g\left(\bar{Y}_{s}, \widehat{Y}_{s}\right) d W(s)\right|^{2} \\
& \quad+5 E\left|\int_{n h}^{t} h\left(\bar{Y}_{s}, \widehat{Y}_{s}, \bar{\gamma}(s)\right) d N(s)\right|^{2} .
\end{aligned}
$$

Now, the Cauchy-Schwarz inequality and Assumptions 2-7 give

$$
\begin{aligned}
& E\left[\sup _{t \in[0, T]}\left|Q_{t}-\bar{Y}_{t}\right|^{2}\right] \\
& \leq 5 h E\left[\sup _{t \in[0, T]} \int_{k h}^{t}\left|\frac{\partial Q_{s}}{\partial a}\right|^{2} d s\right] \\
& \quad+10 h \bar{\alpha}^{2} E\left[\sup _{t \in[0, T]} \int_{n h}^{t}\left(\left|\bar{Y}_{s}\right|^{2}+\left|\bar{Z}_{s}\right|^{2}\right) d s\right] \\
& \quad+10 h E\left[\sup _{t \in[0, T]} \int_{n h}^{t}\left(\left|f\left(\bar{Y}_{s}, \widehat{Y}_{s}\right)\right|^{2}+\left|f\left(\bar{Z}_{s}, \widehat{Z}_{s}\right)\right|^{2}\right) d s\right]
\end{aligned}
$$




$$
\begin{aligned}
& +5 E\left[\sup _{t \in[0, T]}\left|\int_{n h}^{t} g\left(\bar{Y}_{s}, \widehat{Y}_{s}\right) d W(s)\right|^{2}\right] \\
& +10 E\left[\sup _{t \in[0, T]}\left|\int_{n h}^{t} h\left(\bar{Y}_{s}, \widehat{Y}_{s}, \bar{\gamma}(s)\right) d \bar{N}(s)\right|^{2}\right] \\
& +10 E\left[\sup _{t \in[0, T]}\left|\lambda \int_{n h}^{t} h\left(\bar{Y}_{s}, \widehat{Y}_{s}, \bar{\gamma}(s)\right) d s\right|^{2}\right] \\
& \leq 5 h E\left[\sup _{t \in[0, T]} \int_{k h}^{t}\left|\frac{\partial Q_{s}}{\partial a}\right|^{2} d s\right] \\
& +10 h \bar{\alpha}^{2} E\left[\sup _{t \in[0, T]} \int_{n h}^{t}\left(\left|\bar{Y}_{s}\right|^{2}+\left|\bar{Z}_{s}\right|^{2}\right) d s\right] \\
& +5\left(4 h^{2}+h+2 h^{2} \lambda^{2}+2 k_{8} h\right) K_{d}^{\prime},
\end{aligned}
$$

where $k_{8}$ is a constant. Because the differential operator $\partial / \partial a$ is a bounded linear operator, we obtain

$$
\begin{aligned}
E\left[\sup _{t \in[0, T]}\left|Q_{t}-\bar{Y}_{t}\right|^{2}\right] \\
\leq 5 h^{2} E\left|\frac{\partial Q_{s}}{\partial a}\right|^{2}+20 \bar{\alpha}^{2} h^{2} \sup _{t \in[0, T]} E\left|Q_{t}\right|^{2} \\
\quad+5\left(4 h+1+2 h \lambda^{2}+2 k_{8}\right) h K_{d}^{\prime} \\
\leq C_{1}(d) h .
\end{aligned}
$$

Similarly, the second part of (25) can be obtained.

Lemma 13. Under Assumptions 2-7, for any $t \in[0, T]$,

$$
\begin{aligned}
& E\left[\sup _{t \in[0, T]}\left|Q_{t-\tau(t)}-\widehat{Y}_{t}\right|^{2}\right] \leq C_{2}(d) h^{1 \wedge 2 \gamma}, \\
& E\left[\sup _{t \in[0, T]}\left|Q_{t-\tau(t)}-\widehat{Z}_{t}\right|^{2}\right] \leq C_{4}(d) h^{1 \wedge 2 \gamma},
\end{aligned}
$$

where $C_{2}(d), C_{4}(d)$ are positive constants dependent only on $\theta$, $\lambda$, and $K_{d}^{\prime}$ and independent of $h$.

Proof. For any $t \in[0, T]$, there exists an integer $n$ such that $t \in[n h,(n+1) h)$; then $Q_{t-\tau(t)}-\widehat{Y}_{t}=Q_{t-\tau(t)}-Q_{[(n h-\tau(n h)) / h] h}$. To show the estimate $E\left[\left|Q_{t-\tau(t)}-\widehat{Y}_{t}\right|^{2}\right]$, let us consider the following five possible cases.

(1) If $t-\tau(t) \geq[(n h-\tau(n h)) / h] h \geq 0$, then $t-\tau(t)-[(n h-$ $\tau(n h)) / h] h \leq t-\tau(t)+\tau(n h)-(n-1) h \leq(k+2) h$.
Using the Cauchy-Schwarz inequality, martingale isometries, and (15), we have

$$
\begin{aligned}
& \left|Q_{t-\tau(t)}-\widehat{Y}_{t}\right|^{2} \\
& \leq 5\left|\int_{[(n h-\tau(n h)) / h] h}^{t-\tau(t)} \frac{\partial Q_{s}}{\partial a} d s\right|^{2} \\
& +5\left|\int_{[(n h-\tau(n h)) / h] h}^{t-\tau(t)} \mu(s, a)\left[(1-\theta) \bar{Y}_{s}+\theta \bar{Z}_{s}\right] d s\right|^{2} \\
& +5 \mid \int_{[(n h-\tau(n h)) / h] h}^{t-\tau(t)}\left[(1-\theta) f\left(\bar{Y}_{s}, \widehat{Y}_{s}\right)\right. \\
& \left.+\theta f\left(\bar{Z}_{s}, \widehat{Z}_{s}\right)\right]\left.d s\right|^{2} \\
& +5\left|\int_{[(n h-\tau(n h)) / h] h}^{t-\tau(t)} g\left(\bar{Y}_{s}, \widehat{Y}_{s}\right) d W(s)\right|^{2} \\
& +5\left|\int_{[(n h-\tau(n h)) / h] h}^{t-\tau(t)} h\left(\bar{Y}_{s}, \widehat{Y}_{s}, \bar{\gamma}(s)\right) d N(s)\right|^{2} \\
& \leq 5(k+2)^{2} h^{2}\left|\frac{\partial Q_{s}}{\partial a}\right|^{2} \\
& +10 \bar{\alpha}^{2}(k+2) h \int_{[(n h-\tau(n h)) / h] h}^{t-\tau(t)}\left(\left|\bar{Y}_{s}\right|^{2}+\left|\bar{Z}_{s}\right|^{2}\right) d s \\
& +10(k+2) h \\
& \times \int_{[(n h-\tau(n h)) / h] h}^{t-\tau(t)}\left(\left|f\left(\bar{Y}_{s}, \widehat{Y}_{s}\right)\right|^{2}+\left|f\left(\bar{Z}_{s}, \widehat{Z}_{s}\right)\right|^{2}\right) d s \\
& +5 \int_{[(n h-\tau(n h)) / h] h}^{t-\tau(t)}\left|g\left(\bar{Y}_{s}, \widehat{Y}_{s}\right)\right|^{2} d s \\
& +\left(10 \lambda+10 \lambda^{2}(k+2) h\right) \\
& \times \int_{[(n h-\tau(n h)) / h] h}^{t-\tau(t)}\left|h\left(\bar{Y}_{s}, \widehat{Y}_{s}, \bar{\gamma}(s)\right)\right|^{2} d s .
\end{aligned}
$$

Hence,

$$
\begin{aligned}
& E\left[\sup _{t \in[0, T]}\left|Q_{t-\tau(t)}-\widehat{Y}_{t}\right|^{2}\right] \\
& \leq 5(k+2)^{2} h^{2} E\left|\frac{\partial Q_{s}}{\partial a}\right|^{2}+20 \bar{\alpha}^{2}(k+2)^{2} h^{2} \sup _{t \in[0, T]} E\left|Q_{t}\right|^{2} \\
& \quad+5\left(2 \lambda+2 \lambda^{2}(k+2) h+1+4(k+2) h\right)(k+2) h K_{d}^{\prime} \\
& \leq C_{2}(d) h .
\end{aligned}
$$

(2) If $[(n h-\tau(n h)) / h] h \geq t-\tau(t) \geq 0$, then $[(n h-$ $\tau(n h)) / h] h-(t-\tau(t)) \leq n h-\tau(n h)-(t-\tau(t)) \leq k h$. 
Using the Cauchy-Schwarz inequality, martingale isometries, and (15), we have

$$
\begin{aligned}
& \left|Q_{t-\tau(t)}-\widehat{Y}_{t}\right|^{2} \\
& \leq 5\left|\int_{t-\tau(t)}^{[(n h-\tau(n h)) / h] h} \frac{\partial Q_{s}}{\partial a} d s\right|^{2} \\
& +5\left|\int_{t-\tau(t)}^{[(n h-\tau(n h)) / h] h} \mu(s, a)\left[(1-\theta) \bar{Y}_{s}+\theta \bar{Z}_{s}\right] d s\right|^{2} \\
& +5\left|\int_{t-\tau(t)}^{[(n h-\tau(n h)) / h] h}\left[(1-\theta) f\left(\bar{Y}_{s}, \widehat{Y}_{s}\right)+\theta f\left(\bar{Z}_{s}, \widehat{Z}_{s}\right)\right] d s\right|^{2} \\
& +5\left|\int_{t-\tau(t)}^{[(n h-\tau(n h)) / h] h} g\left(\bar{Y}_{s}, \widehat{Y}_{s}\right) d W(s)\right|^{2} \\
& +5\left|\int_{t-\tau(t)}^{[(n h-\tau(n h)) / h] h} h\left(\bar{Y}_{s}, \widehat{Y}_{s}, \bar{\gamma}(s)\right) d N(s)\right|^{2} \\
& \leq 5 k^{2} h^{2}\left|\frac{\partial Q_{s}}{\partial a}\right|^{2} \\
& +10 \bar{\alpha}^{2} k h \int_{t-\tau(t)}^{[(n h-\tau(n h)) / h] h}\left(\left|\bar{Y}_{s}\right|^{2}+\left|\bar{Z}_{s}\right|^{2}\right) d s \\
& +10 k h \int_{t-\tau(t)}^{[(n h-\tau(n h)) / h] h}\left(\left|f\left(\bar{Y}_{s}, \widehat{Y}_{s}\right)\right|^{2}+\left|f\left(\bar{Z}_{s}, \widehat{Z}_{s}\right)\right|^{2}\right) d s \\
& +5 E \int_{t-\tau(t)}^{[(n h-\tau(n h)) / h] h}\left|g\left(\bar{Y}_{s}, \widehat{Y}_{s}\right)\right|^{2} d s \\
& +\left(10 \lambda+10 \lambda^{2} k h\right) \int_{[(n h-\tau(n h)) / h] h}^{t-\tau(t)}\left|h\left(\bar{Y}_{s}, \widehat{Y}_{s}, \bar{\gamma}(s)\right)\right|^{2} d s
\end{aligned}
$$

Hence,

$$
\begin{aligned}
E\left[\sup _{t \in[0, T]}\left|Q_{t-\tau(t)}-\widehat{Y}_{t}\right|^{2}\right] \\
\leq 5 k^{2} h^{2} E\left|\frac{\partial Q_{s}}{\partial a}\right|^{2}+20 \bar{\alpha}^{2} k^{2} h^{2} \sup _{t \in[0, T]} E\left|Q_{t}\right|^{2} \\
\quad+5\left(2 \lambda+2 \lambda^{2} k h+1+4 k h\right) k h K^{\prime} \\
\leq C_{2}(d) h .
\end{aligned}
$$

(3) If $0 \geq t-\tau(t) \geq[(n h-\tau(n h)) / h] h$ or $0 \geq[(n h-$ $\tau(n h)) / h] h \geq t-\tau(t)$, then $\mid t-\tau(t)-[(n h-$ $\tau(n h)) / h] h \mid \leq(k+2) h$. So we get, by Assumption 5 on $\psi$,

$$
\begin{aligned}
& E\left[\left|Q_{t-\tau(t)}-\widehat{Y}_{t}\right|^{2}\right] \\
& \quad=E|\psi(t-\tau(t))-\psi([(n h-\tau(n h)) / h] h)|^{2}
\end{aligned}
$$

$$
\begin{aligned}
& \leq K^{\prime}\left|s-\tau(s)-\left[\frac{(n h-\tau(n h))}{h}\right] h\right|^{2} \\
& \leq K^{\prime}(k+2)^{2 \gamma} h^{2 \gamma} .
\end{aligned}
$$

(4) If $t-\tau(t) \geq 0 \geq[(n h-\tau(n h)) / h] h$, then $t-\tau(t) \leq$ $t-\tau(t)-[(n h-\tau(n h)) / h] h \leq(k+2) h$ and $-[(n h-$ $\tau(n h)) / h] h \leq t-\tau(t)-[(n h-\tau(n h)) / h] h \leq(k+2) h$. We have, by Assumption 5 on $\psi$,

$$
\begin{aligned}
& \left|Q_{t-\tau(t)}-\widehat{Y}_{t}\right|^{2} \\
& =\left|Q_{t-\tau(t)}-\psi\left(\left[\frac{(n h-\tau(n h))}{h}\right] h\right)\right|^{2} \\
& =\left|Q_{t-\tau(t)}-\psi(0)+\psi(0)-\psi\left(\left[\frac{(n h-\tau(n h))}{h}\right] h\right)\right|^{2} \\
& \leq 2\left|Q_{t-\tau(t)}-\psi(0)\right|^{2} \\
& +2\left|\psi(0)-\psi\left(\left[\frac{(n h-\tau(n h))}{h}\right] h\right)\right|^{2} \\
& =2\left|Q_{t-\tau(t)}-Q_{0}\right|^{2}+2\left|\psi(0)-\psi\left(\left[\frac{(n h-\tau(n h))}{h}\right] h\right)\right|^{2} \\
& \leq 10\left|\int_{0}^{t-\tau(t)} \frac{\partial Q_{s}}{\partial a} d s\right|^{2} \\
& +10\left|(1-\theta) \int_{0}^{t-\tau(t)} \mu(s, a)\left[(1-\theta) \bar{Y}_{s}+\theta \bar{Z}_{s}\right] d s\right|^{2} \\
& +10\left|\int_{0}^{t-\tau(t)}\left[(1-\theta) f\left(\bar{Y}_{s}, \widehat{Y}_{s}\right)+\theta f\left(\bar{Z}_{s}, \widehat{Z}_{s}\right)\right] d s\right|^{2} \\
& \quad+\left.1 \frac{\left(n h-\tau\left(\bar{Y}_{s}, \widehat{Y}_{s}\right) d W(s) \mid\right.}{h}\right|^{2}
\end{aligned}
$$

Hence,

$$
\begin{aligned}
& E\left[\sup _{t \in[0, T]}\left|Q_{t-\tau(t)}-\widehat{Y}_{t}\right|^{2}\right] \\
& \leq 10(k+2)^{2} h^{2} E\left|\frac{\partial Q_{s}}{\partial a}\right|^{2}+20 \bar{\alpha}^{2}(k+2)^{2} h^{2} \sup _{t \in[0, T]} E\left|Q_{t}\right|^{2}
\end{aligned}
$$




$$
\begin{aligned}
& +5\left(2 \lambda+2 \lambda^{2} k h+1+4 k h\right)(k+2) h K^{\prime} \\
& +2 K^{\prime}(k+2)^{2 \gamma} h^{2 \gamma} \\
\leq & C_{2}(d) h^{1 \wedge 2 \gamma} .
\end{aligned}
$$

(5) If $[(n h-\tau(n h)) / h] h \geq 0 \geq t-\tau(t)$, then $[(n h-$ $\tau(n h)) / h] h \leq[(n h-\tau(n h)) / h] h-(t-\tau(t)) \leq(k+2) h$ and $-(t-\tau(t)) \leq[(n h-\tau(n h)) / h] h-(t-\tau(t)) \leq(k+2) h$. We have, by Assumption 5 on $\psi$,

$$
\begin{aligned}
& \left|Q_{t-\tau(t)}-\widehat{Y}_{t}\right|^{2} \\
& =\left|\psi(t-\tau(t))-Q_{[(n h-\tau(n h)) / h] h}\right|^{2} \\
& =\left|\psi(t-\tau(t))-\psi(0)+\psi(0)-Q_{[(n h-\tau(n h)) / h] h}\right|^{2} \\
& \leq 2|\psi(t-\tau(t))-\psi(0)|^{2}+2\left|\psi(0)-Q_{[(n h-\tau(n h)) / h] h}\right|^{2} \\
& =2 K^{\prime}|-(t-\tau(t))|^{2 \gamma}+2\left|Q_{0}-Q_{[(n h-\tau(n h)) / h] h}\right|^{2} \\
& \leq 2 K^{\prime}|-(t-\tau(t))|^{2 \gamma}+10\left|\int_{0}^{[(n h-\tau(n h)) / h] h} \frac{\partial Q_{s}}{\partial a} d s\right|^{2} \\
& \quad+10\left|\int_{0}^{[(n h-\tau(n h)) / h] h} \mu(s, a)\left[(1-\theta) \bar{Y}_{s}+\theta \bar{Z}_{s}\right] d s\right|^{2} \\
& \quad+10\left|\int_{0}^{[(n h-\tau(n h)) / h] h}\left[(1-\theta) f\left(\bar{Y}_{s}, \widehat{Y}_{s}\right)+\theta f\left(\bar{Z}_{s}, \widehat{Z}_{s}\right)\right] d s\right|^{2} \\
& +10\left|\int_{0}^{[(n h-\tau(n h)) / h] h} g\left(\bar{Y}_{s}, \widehat{Y}_{s}\right) d W(s)\right|^{2} \\
& \left.\quad+10 \mid \int_{0}^{[(n h-\tau(n h)) / h] h}, \widehat{Y}_{s}, \bar{\gamma}(s)\right)\left.d N(s)\right|^{2} \cdot
\end{aligned}
$$

Hence,

$$
\begin{aligned}
& E\left[\sup _{t \in[0, T]}\left|Q_{t-\tau(t)}-\hat{Y}_{t}\right|^{2}\right] \\
& \leq 10(k+2)^{2} h^{2} E\left|\frac{\partial Q_{s}}{\partial a}\right|^{2}+20 \bar{\alpha}^{2}(k+2)^{2} h^{2} \sup _{t \in[0, T]} E\left|Q_{t}\right|^{2} \\
& \quad+5\left(2 \lambda+2 \lambda^{2} k h+1+4 k h\right)(k+2) h K^{\prime} \\
& \quad+2 K^{\prime}(k+2)^{2 \gamma} h^{2 \gamma} \\
& \leq C_{2}(d) h^{1 \wedge 2 \gamma} .
\end{aligned}
$$

Combining these different cases, we get

$$
E\left[\sup _{t \in[0, T]}\left|Q_{t-\tau(t)}-\widehat{Y}_{t}\right|^{2}\right] \leq C_{2}(d) h^{1 \wedge 2 \gamma}
$$

Similarly, we have

$$
E\left[\sup _{t \in[0, T]}\left|Q_{t-\tau(t)}-\widehat{Z}_{t}\right|^{2}\right] \leq C_{4}(d) h^{1 \wedge 2 \gamma}
$$

Lemma 14. There exists a constant $C$ for any $t \in[0, T]$ and $E\left[\left|\gamma_{i}\right|^{p}\right] \leq C$ such that

$$
E\left[\int_{0}^{t}|\gamma(s)-\bar{\gamma}(s)|^{2} d s\right] \leq C h^{1-2 / p} .
$$

Proof. The proof is basically similar to that of Theorem 3.4 in [15], and we thus omit it here.

We are now in a position to prove the main convergence results.

Theorem 15. If Assumptions 2-7 hold, then the semi-implicit Euler approximate solutions converge to the exact solutions of (1) in the mean-square sense; that is,

$$
E\left[\sup _{0 \leq t \leq T}\left|Q_{t \wedge v_{n}}-K_{t \wedge v_{n}}\right|^{2}\right] \leq C_{d} h^{(1-2 / p) \wedge 2 \gamma}
$$

where $C_{d}$ is a positive constant dependent only on $\theta, \lambda$, and $K_{d}$ and $T$ and independent of $h$.

Proof. Subtraction of (6) and (9) gives

$$
\begin{aligned}
& K_{t}-Q_{t} \\
& =-\int_{0}^{t} \frac{\partial\left(K_{s}-Q_{s}\right)}{\partial a} d s \\
& \quad-\int_{0}^{t} \mu(s, a)\left[(1-\theta)\left(K_{s}-\bar{Y}_{s}\right)+\theta\left(K_{s}-\bar{Z}_{s}\right)\right] d s \\
& +\int_{0}^{t}(1-\theta)\left[\left(f\left(K_{s}, K_{s-\tau(s)}\right)-f\left(\bar{Y}_{s}, \widehat{Y}_{s}\right)\right)\right. \\
& \quad+\int_{0}^{t} g\left(K_{s}, K_{s-\tau(s)}\right)-g\left(\bar{Y}_{s}, \widehat{Y}_{s}\right) d W(s) \\
& \quad+\int_{0}^{t} h\left(K_{s}, K_{s-\tau(s)}, \gamma(s)\right)-h\left(\bar{Y}_{s}, \widehat{Y}_{s}, \bar{\gamma}(s)\right) d N(s) .
\end{aligned}
$$


Therefore using the generalized Itô formula, along with the Cauchy-Schwarz inequality and Assumptions 2-7, yields

$$
\begin{aligned}
& \left|K_{t}-Q_{t}\right|^{2} \\
& =-2 \int_{0}^{t}\left\langle K_{s}-Q_{s}, \frac{\partial\left(K_{s}-Q_{s}\right)}{\partial a}\right\rangle d s \\
& \quad-2 \int_{0}^{t}\left(K_{s}-Q_{s},\right. \\
& \left.\quad \mu(s, a)\left[(1-\theta)\left(K_{s}-\bar{Y}_{s}\right)+\theta\left(K_{s}-\bar{Z}_{s}\right)\right] d s\right) \\
& +2 \int_{0}^{t}\left(K_{s}-Q_{s},\left[(1-\theta)\left(f\left(K_{s}, K_{s-\tau(s)}\right)-f\left(\bar{Y}_{s}, \widehat{Y}_{s}\right)\right)\right.\right. \\
& \quad+2 \int_{0}^{t}\left(K_{s}-Q_{s}, g\left(K_{s}, K_{s-\tau(s)}\right)-g\left(\bar{Y}_{s}, \widehat{Y}_{s}\right) d W(s)\right) \\
& +\int_{0}^{t}\left\|g\left(K_{s}, K_{s-\tau(s)}\right)-g\left(\bar{Y}_{s}, \widehat{Y}_{s}\right)\right\|_{2}^{2} d s \\
& +2 \int_{0}^{t}\left(K_{s}-Q_{s},\right.
\end{aligned}
$$$$
\left.\left(h\left(K_{s}, K_{s-\tau(s)}, \gamma(s)\right)-h\left(\bar{Y}_{s}, \widehat{Y}_{s}, \bar{\gamma}(s)\right)\right)\right) d N(s)
$$$$
+\int_{0}^{t}\left|h\left(K_{s}, K_{s-\tau(s)}, \gamma(s)\right)-h\left(\bar{Y}_{s}, \widehat{Y}_{s}, \bar{\gamma}(s)\right)\right|^{2} d N(s)
$$$$
\leq\left(A^{2} F_{1}^{2} \eta^{2}+9+9 \bar{\alpha}\right) \int_{0}^{t}\left|K_{s}-Q_{s}\right|^{2} d s
$$$$
+4\left(\bar{\alpha}+K_{d}\right) \int_{0}^{t}\left[\left|Q_{s}-\bar{Y}_{s}\right|^{2}+\left|Q_{s}-\bar{Z}_{s}\right|^{2}\right] d s
$$$$
+4 K_{d} \int_{0}^{t}\left|K_{s-\tau}-Q_{s-\tau}\right|^{2} d s
$$$$
+4 K_{d} \int_{0}^{t}\left[\left|Q_{s-\tau}-\widehat{Y}_{s}\right|^{2}+\left|Q_{s-\tau}-\widehat{Z}_{s}\right|^{2}\right] d s
$$$$
+2 \int_{0}^{t}\left(K_{s}-Q_{s}, g\left(K_{s}, K_{s-\tau(s)}\right)-g\left(\bar{Y}_{s}, \widehat{Y}_{s}\right) d W(s)\right)
$$$$
+\int_{0}^{t}\left\|g\left(K_{s}, K_{s-\tau(s)}\right)-g\left(\bar{Y}_{s}, \widehat{Y}_{s}\right)\right\|_{2}^{2} d s
$$$$
+2 \lambda \int_{0}^{t}\left(K_{s}-Q_{s}\right.
$$$$
\left.\left(h\left(K_{s}, K_{s-\tau(s)}, \gamma(s)\right)-h\left(\bar{Y}_{s}, \widehat{Y}_{s}, \bar{\gamma}(s)\right)\right) d s\right)
$$$$
+2 \int_{0}^{t}\left(K_{s}-Q_{s},\right.
$$$$
\left.\left(h\left(K_{s}, K_{s-\tau(s)}, \gamma(s)\right)-h\left(\bar{Y}_{s}, \widehat{Y}_{s}, \bar{\gamma}(s)\right)\right) d \bar{N}(s)\right)
$$

$$
\begin{aligned}
& +\lambda \int_{0}^{t}\left|h\left(K_{s}, K_{s-\tau(s)}, \gamma(s)\right)-h\left(\bar{Y}_{s}, \widehat{Y}_{s}, \bar{\gamma}(s)\right)\right|^{2} d s \\
& +\int_{0}^{t}\left|h\left(K_{s}, K_{s-\tau(s)}, \gamma(s)\right)-h\left(\bar{Y}_{s}, \widehat{Y}_{s}, \bar{\gamma}(s)\right)\right|^{2} d \bar{N}(s) .
\end{aligned}
$$

By Burkholder-Davis-Gundy inequality, we have

$$
\begin{aligned}
& 2 E \sup _{s \in[0, t]} \int_{0}^{s \wedge v_{n}}\left(K_{u}-Q_{u}, g\left(K_{u}, K_{u-\tau(u)}\right)-g\left(\bar{Y}_{u}, \widehat{Y}_{u}\right) d W(u)\right) \\
& \leq 2 k_{9} \int_{0}^{t \wedge v_{n}} E\left(\left|K_{s}-Q_{s}\right|^{2}+\left|Q_{s}-\bar{Y}_{s}\right|^{2}\right. \\
& \left.+\left|K_{s-\tau(s)}-Q_{s-\tau(s)}\right|^{2}+\left|Q_{s-\tau(s)}-\widehat{Y}_{s}\right|^{2}\right) d s \\
& +\frac{1}{6} E\left[\sup _{0 \leq s \leq t}\left|K_{s \wedge v_{n}}-Q_{s \wedge v_{n}}\right|^{2}\right] \text {, } \\
& 2 E\left[\operatorname { s u p } _ { s \in [ 0 , t ] } \int _ { 0 } ^ { s \wedge v _ { n } } \left(K_{u}-Q_{u},\left(h\left(K_{u}, K_{u-\tau(u)}, \gamma(u)\right)\right.\right.\right. \\
& \left.\left.\left.-h\left(\bar{Y}_{u}, \widehat{Y}_{u}, \bar{\gamma}(u)\right)\right) d \bar{N}(u)\right)\right] \\
& \leq \frac{1}{6} E\left[\sup _{0 \leq s \leq t}\left|K_{s \wedge v_{n}}-Q_{s \wedge v_{n}}\right|^{2}\right] \\
& +k_{11} \int_{0}^{t \wedge v_{n}} E\left(|\gamma(s)-\bar{\gamma}(s)|^{2}\right) d s \\
& +2 k_{10} \int_{0}^{t \wedge v_{n}} E\left(\left|K_{s}-Q_{s}\right|^{2}\right. \\
& +\left|Q_{s}-\bar{Y}_{s}\right|^{2}+\left|K_{s-\tau(s)}-Q_{s-\tau(s)}\right|^{2} \\
& \left.+\left|Q_{s-\tau(s)}-\widehat{Y}_{s}\right|^{2}\right) d s, \\
& E\left[\int_{0}^{s \wedge v_{n}}\left|h\left(K_{u}, K_{u-\tau(u)}, \gamma(u)\right)-h\left(\bar{Y}_{u}, \widehat{Y}_{u}, \bar{\gamma}(u)\right)\right|^{2} d \bar{N}(u)\right] \\
& \leq \frac{1}{6} E\left[\sup _{0 \leq s \leq t}\left|K_{s \wedge v_{n}}-Q_{s \wedge v_{n}}\right|^{2}\right] \\
& +\frac{1}{6} E\left[\sup _{0 \leq s \leq t}\left|Q_{s \wedge v_{n}}-\bar{Y}_{s \wedge v_{n}}\right|^{2}\right] \\
& +\frac{1}{6} E\left[\sup _{0 \leq u \leq t}\left|K_{u \wedge v_{n}}-Q_{u \wedge v_{n}}\right|^{2}\right] \\
& +\frac{1}{6} E\left[\sup _{0 \leq u \leq t}\left|Q_{u \wedge v_{n}}-\widehat{Y}_{s}\right|^{2}\right] \\
& +k_{13} \int_{0}^{t \wedge v} E\left(|\gamma(s)-\bar{\gamma}(s)|^{2}\right) d s \\
& +2 k_{12} \int_{0}^{t \wedge v_{n}} E\left(\left|K_{s}-Q_{s}\right|^{2}\right.
\end{aligned}
$$




$$
\begin{aligned}
& +\left|Q_{s}-\bar{Y}_{s}\right|^{2}+\left|K_{s-\tau(s)}-Q_{s-\tau(s)}\right|^{2} \\
& \left.+\left|Q_{s-\tau(s)}-\widehat{Y}_{s}\right|^{2}\right) d s,
\end{aligned}
$$

$2 \lambda E \sup _{s \in[0, t]} \int_{0}^{s \wedge v_{n}}\left(K_{u}-Q_{u}\right.$,

$$
\left.\left(h\left(K_{u}, K_{u-\tau(u)}, \gamma(u)\right)-h\left(\bar{Y}_{u}, \widehat{Y}_{u}, \bar{\gamma}(u)\right)\right) d u\right)
$$

$\leq \lambda \int_{0}^{t \wedge v_{n}} E\left|K_{s}-Q_{s}\right|^{2} d s$

$+\lambda K \int_{0}^{t \wedge v_{n}} E\left(|\gamma(s)-\bar{\gamma}(s)|^{2}\right) d s$

$+2 \lambda K_{d} E \int_{0}^{t \wedge v_{n}}\left(\left|K_{s}-Q_{s}\right|^{2}\right.$

$$
\begin{aligned}
& +\left|Q_{s}-\bar{Y}_{s}\right|^{2}+\left|K_{s-\tau(s)}-Q_{s-\tau(s)}\right|^{2} \\
& \left.+\left|Q_{s-\tau(s)}-\widehat{Y}_{s}\right|^{2}\right) d s,
\end{aligned}
$$

$\lambda \int_{0}^{t \wedge v_{n}}\left|h\left(K_{s}, K_{s-\tau(s)}, \gamma(s)\right)-h\left(\bar{Y}_{s}, \widehat{Y}_{s}, \bar{\gamma}(s)\right)\right|^{2} d s$

$\leq 2 \lambda K_{d} \int_{0}^{t \wedge v_{n}}\left(\left|K_{s}-Q_{s}\right|^{2}\right.$

$$
\begin{aligned}
& +\left|Q_{s}-\bar{Y}_{s}\right|^{2}+\left|K_{s-\tau(s)}-Q_{s-\tau(s)}\right|^{2} \\
& \left.+\left|Q_{s-\tau(s)}-\widehat{Y}_{s}\right|^{2}\right) d s
\end{aligned}
$$

$$
+\lambda K \int_{0}^{t \wedge v_{n}}\left(|\gamma(s)-\bar{\gamma}(s)|^{2}\right) d s
$$

$$
\int_{0}^{t \wedge v_{n}}\left\|g\left(K_{s}, K_{s-\tau(s)}\right)-g\left(\bar{Y}_{s}, \widehat{Y}_{s}\right)\right\|_{2}^{2} d s
$$$$
\leq K_{d} \int_{0}^{t \wedge v_{n}}\left(\left|K_{s}-\bar{Y}_{s}\right|^{2}+\left|K_{s-\tau(s)}-\widehat{Y}_{s}\right|^{2}\right) d s
$$$$
\leq 2 K_{d} \int_{0}^{t \wedge v_{n}}\left(\left|K_{s}-Q_{s}\right|^{2}\right.
$$

$$
\begin{aligned}
& +\left|Q_{s}-\bar{Y}_{s}\right|^{2}+\left|K_{s-\tau(s)}-Q_{s-\tau(s)}\right|^{2} \\
& \left.+\left|Q_{s-\tau(s)}-\widehat{Y}_{s}\right|^{2}\right) d s
\end{aligned}
$$

where $k_{9}, k_{10}, k_{11}, k_{12}$, and $k_{13}$ are positive constants. Let $k=$ $\max \left\{k_{9}, k_{10}, k_{11}, k_{12}, k_{13}\right\}$; inserting (46) into (45) we obtain

$$
\begin{aligned}
& E\left[\sup _{s \in[0, T]}\left|K_{s \wedge v_{n}}-Q_{s \wedge v_{n}}\right|^{2}\right] \\
& \leq\left(A^{2} F_{1}^{2} \eta^{2}+9+\lambda+9 \bar{\alpha}\right)
\end{aligned}
$$

$$
\begin{aligned}
& \times \int_{0}^{t \wedge v_{n}} E \sup _{u \in[0, s]}\left|K_{u \wedge v_{n}}-Q_{u \wedge v_{n}}\right|^{2} d s \\
& +4\left(\bar{\alpha}+K_{d}\right) E \int_{0}^{t \wedge v_{n}}\left[\left|Q_{s}-\bar{Y}_{s}\right|^{2}+\left|Q_{s}-\bar{Z}_{s}\right|^{2}\right] d s \\
& +4 K_{d} E \int_{0}^{t \wedge v_{n}}\left|K_{s-\tau}-Q_{s-\tau}\right|^{2} d s \\
& +4 K_{d} E \int_{0}^{t \wedge v_{n}}\left[\left|Q_{s-\tau}-\widehat{Y}_{s}\right|^{2}+\left|Q_{s-\tau}-\widehat{Z}_{s}\right|^{2}\right] d s \\
& +\frac{2}{3} E\left[\sup _{0 \leq s \leq t}\left|K_{s \wedge v_{n}}-Q_{s \wedge v_{n}}\right|^{2}\right] \\
& +\frac{1}{6} E\left[\sup _{0 \leq s \leq t}\left|Q_{s \wedge v_{n}}-\bar{Y}_{s \wedge v_{n}}\right|^{2}\right] \\
& +\frac{1}{6} E\left[\sup _{0 \leq u \leq t}\left|Q_{u \wedge v_{n}}-\widehat{Y}_{s}\right|^{2}\right] \\
& +\left(2 K_{d}+4 \lambda K_{d}+6 k\right) \\
& \times \int_{0}^{t \wedge v_{n}} E\left(\left|K_{s}-Q_{s}\right|^{2}\right. \\
& +\left|Q_{s}-\bar{Y}_{s}\right|^{2}+\left|K_{s-\tau(s)}-Q_{s-\tau(s)}\right|^{2} \\
& \left.+\left|Q_{s-\tau(s)}-\widehat{Y}_{s}\right|^{2}\right) d s, \\
& +(2 \lambda K+2 k) \int_{0}^{t \wedge v_{n}} E\left(|\gamma(s)-\bar{\gamma}(s)|^{2}\right) d s .
\end{aligned}
$$

Hence,

$$
\begin{aligned}
& E\left[\sup _{s \in[0, T]}\left|K_{s \wedge v_{n}}-Q_{s \wedge v_{n}}\right|^{2}\right] \\
& \leq 3\left(A^{2} F_{1}^{2} \eta^{2}+9+\lambda+9 \bar{\alpha}\right) \\
& \quad \times \int_{0}^{t \wedge v_{n}} E \sup _{u \in[0, s]}\left|K_{u \wedge v_{n}}-Q_{u \wedge v_{n}}\right|^{2} d s \\
& +12 h^{1 \wedge 2 \gamma} T\left[\left(\bar{\alpha}+K_{d}\right)\left(C_{1}(d)+C_{3}(d)\right)\right. \\
& \left.\quad+6(k+\lambda K) C h^{1-2 / p}\left(C_{2}(d)+C_{4}(d)\right)\right] \\
& +\left[6\left(3 k+2 \lambda K_{d}+K_{d}\right) T+1\right]\left(C_{1}(d)+C_{2}(d)\right) h^{1 \wedge 2 \gamma} \\
& +12\left(3 k+2 \lambda K_{d}+2 K_{d}\right) \\
& \quad \times \int_{0}^{t \wedge v_{n}} E\left[\sup _{0 \leq u \leq s}\left|K_{u \wedge v_{n}}-Q_{u \wedge v_{n}}\right|^{2}\right] d s
\end{aligned}
$$




$$
\begin{aligned}
\leq & C_{5}(d) h^{(1-2 / p) \wedge 2 \gamma} \\
& +C_{6}(d) \int_{0}^{t \wedge v_{n}} E\left[\sup _{0 \leq u \leq s}\left|K_{u \wedge v_{n}}-Q_{u \wedge v_{n}}\right|^{2}\right] d s .
\end{aligned}
$$

Applying Gronwall's inequality, we obtain a bound of the form

$$
E\left[\sup _{s \in[0, T]}\left|K_{s \wedge v_{n}}-Q_{s \wedge v_{n}}\right|^{2}\right] \leq C_{d} h^{(1-2 / p) \wedge 2 \gamma}
$$

where $C_{d}=C_{5}(d) e^{C_{6}(d) T}$.

Theorem 16. Under Assumptions 2-7 and let $0<\theta<1$, then

$$
E\left[\sup _{0 \leq t \leq T}\left|Q_{t}-K_{t}\right|^{2}\right] \leq C_{d}^{\prime} h^{(1-2 / p) \wedge 2 \gamma} .
$$

Proof. Let $Z_{t}=Q_{t}-K_{t}$, it is easy to see that

$$
\begin{aligned}
& E\left[\sup _{0 \leq t \leq T}\left|Z_{t}\right|^{2}\right] \\
& =E\left[\sup _{0 \leq t \leq T}\left|Z_{t}\right|^{2} 1_{\left\{\sigma_{n}>T, \rho_{n}>T\right\}}\right]+E\left[\sup _{0 \leq t \leq T}\left|Z_{t}\right|^{2} 1_{\left\{\sigma_{n} \leq T \text { or } \rho_{n} \leq T\right\}}\right] \\
& =E\left[\sup _{0 \leq t \leq T}\left|Z_{t}\right|^{2} 1_{\left\{v_{n}>T\right\}}\right]+E\left[\sup _{0 \leq t \leq T}\left|Z_{t}\right|^{2} 1_{\left\{\sigma_{n} \leq T \text { or } \rho_{n} \leq T\right\}}\right] \\
& \leq E\left[\sup _{0 \leq t \leq T}\left|Z_{t}\right|^{2} 1_{\left\{t \wedge v_{n}\right\}}\right]+E\left[\sup _{0 \leq t \leq T}\left|Z_{t}\right|^{2} 1_{\left\{\sigma_{n} \leq T \text { or } \rho_{n} \leq T\right\}}\right] .
\end{aligned}
$$

By Young's inequality $x y \leq x^{p} / p+y^{q} / q, 1 / p+1 / q=1$, for any $a, b, p, q$, and $\delta>0$, we have

$$
a b \leq a \delta^{1 / p} \frac{b}{\delta^{1 / p}} \leq \frac{\left(a \delta^{1 / p}\right)^{p}}{p}+\frac{b^{q}}{q \delta^{q / p}}=\frac{a^{p} \delta}{p}+\frac{b^{q}}{q \delta^{q / p}} .
$$

Let $p=2, \delta=h>0$; we have

$$
\begin{aligned}
& E\left[\sup _{0 \leq t \leq T}\left|Z_{t}\right|^{2} 1_{\left\{\sigma_{n} \leq T \text { or } \rho_{n} \leq T\right\}}\right] \\
& \leq \frac{h}{2} E\left[\sup _{0 \leq t \leq T}\left|Z_{t}\right|^{4}\right]+\frac{1}{2 h} P\left\{\sigma_{n} \leq T \text { or } \rho_{n} \leq T\right\} .
\end{aligned}
$$

Note

$$
\begin{aligned}
& E\left[\sup _{0 \leq t \leq T}\left|Z_{t}\right|^{4}\right] \leq 8\left(E\left[\sup _{0 \leq t \leq T}\left|K_{t}\right|^{4}\right]+E\left[\sup _{0 \leq t \leq T}\left|Q_{t}\right|^{4}\right]\right) \\
& \leq 8\left(C_{1}+C_{3}\right), \\
& P\left\{\sigma_{n} \leq T\right\}=E\left[1_{\sigma_{n} \leq T} \frac{\left|K_{\sigma_{n}}\right|^{4}}{n^{4}}\right] \leq \frac{C_{1}}{n^{4}}, \\
& P\left\{\rho_{n} \leq T\right\}=E\left[1_{\rho_{n} \leq T} \frac{\left|Q_{\rho_{n}}\right|^{4}}{n^{4}}\right] \leq \frac{C_{3}}{n^{4}} .
\end{aligned}
$$

Then

$$
\begin{aligned}
& E\left[\sup _{0 \leq t \leq T}\left|Z_{t}\right|^{2} 1_{\left\{\sigma_{n} \leq T \text { or } \rho_{n} \leq T\right\}}\right] \\
& \quad \leq 4 h\left(C_{1}+C_{3}\right)+\frac{1}{2 h n^{4}}\left(C_{1}+C_{3}\right) .
\end{aligned}
$$

By Theorem 15, (51) becomes

$$
\begin{aligned}
& E\left[\sup _{0 \leq t \leq T}\left|Z_{t}\right|^{2}\right] \\
& \quad \leq C_{d} h^{(1-2 / p) \wedge 2 \gamma}+4 h\left(C_{1}+C_{3}\right)+\frac{1}{2 h n^{4}}\left(C_{1}+C_{3}\right) .
\end{aligned}
$$

Let $n \geq\left(2 h^{2}\right)^{-1 / 4}$; then

$$
\begin{aligned}
E\left[\sup _{0 \leq t \leq T}\left|Z_{t}\right|^{2}\right] & \leq C_{d} h^{(1-2 / p) \wedge 2 \gamma}+5 h\left(C_{1}+C_{3}\right) \\
& =C_{d}^{\prime} h^{(1-2 / p) \wedge 2 \gamma} .
\end{aligned}
$$

The proof is completed.

Theorem 17. Under Assumptions 2-7 and there is a constant $C$ such that $E\left[\left|\gamma_{i}\right|^{p}\right] \leq C$ for some $p>2$, the numerical approximate solution (7) will converge to the exact solution to (1) in the sense

$$
\lim _{h \rightarrow 0} E\left[\sup _{0 \leq t \leq T}\left|Q_{t}-K_{t}\right|^{2}\right]=0 .
$$

Proof. The proof is easily deduced from Theorem 16.

\section{Conflict of Interests}

The authors declare that there is no conflict of interests regarding the publication of this paper.

\section{Acknowledgments}

This paper was partially supported by The National Natural Science Foundation (61374040), Key Discipline of Shanghai (S30501), Scientific Innovation Program (13ZZ115), and Graduate Innovation Program of Shanghai (54-13-302-102, JWCXSL1301). 


\section{References}

[1] L. Arnold, Stochastic Differential Equations, John Wiley \& Sons, New York, NY, USA, 1972.

[2] P. Kloeden and E. Platen, Numerical Solution of Stochastic Differential Equations, Springer, Berlin, Germany, 1992.

[3] X. Mao, Stochastic Differential Equations and Applications, Horwood, New York, NY, USA, 1997.

[4] E. Platen, "An introduction to numerical methods for stochastic differential equations," Acta Numerica, vol. 8, pp. 197-246, 1999.

[5] E. Platen and N. Bruti-Liberati, Numerical Solution of Stochastic Differential Equations with Jumps in Finance, Springer, Berlin, Germany, 2010.

[6] Y. Maghsoodi, "Mean square efficient numerical solution of jump-diffusion stochastic differential equations," Sankhya: The Indian Journal of Statistics A, vol. 58, no. 1, pp. 25-47, 1996.

[7] W.-J. Ma, Q.-M. Zhang, and C.-Z. Han, "Numerical analysis for stochastic age-dependent population equations with fractional Brownian motion," Communications in Nonlinear Science and Numerical Simulation, vol. 17, no. 4, pp. 1884-1893, 2012.

[8] Q. Zhang, W. Pang, and P. Leung, "Exponential stability of numerical solutions for a class of stochastic age-dependent capital system with Poisson jumps," Journal of Computational and Applied Mathematics, vol. 235, no. 12, pp. 3369-3377, 2011.

[9] Q. Zhang and A. Rathinasamy, "Convergence of numerical solutions for a class of stochastic age-dependent capital system with random jump magnitudes," Applied Mathematics and Computation, vol. 219, no. 14, pp. 7297-7305, 2013.

[10] R. Li, H. Meng, and Y. Dai, "Convergence of numerical solutions to stochastic delay differential equations with jumps," Applied Mathematics and Computation, vol. 172, no. 1, pp. 584-602, 2006.

[11] L. Wang, C. Mei, and H. Xue, "The semi-implicit Euler method for stochastic differential delay equations with jumps," Applied Mathematics and Computation, vol. 192, no. 2, pp. 567-578, 2007.

[12] E. Buckwar, "Introduction to the numerical analysis of stochastic delay differential equations," Journal of Computational and Applied Mathematics, vol. 125, no. 1-2, pp. 297-307, 2000.

[13] U. Küchler and E. Platen, "Strong discrete time approximation of stochastic differential equations with time delay," Mathematics and Computers in Simulation, vol. 54, no. 1-3, pp. 189-205, 2000.

[14] X. Mao and S. Sabanis, "Numerical solutions of stochastic differential delay equations under local Lipschitz condition," Journal of Computational and Applied Mathematics, vol. 151, no. 1, pp. 215-227, 2003.

[15] G. D. Chalmers and D. J. Higham, "Convergence and stability analysis for implicit simulations of stochastic differential equations with random jump magnitudes," Discrete and Continuous Dynamical Systems B, vol. 9, no. 1, pp. 47-64, 2008.

[16] Q. Zhang, "Convergence of numerical solutions for a class of stochastic age-dependent capital system with Markovian switching," Economic Modelling, vol. 28, no. 3, pp. 1195-1201, 2011. 


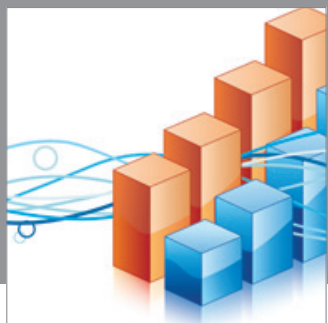

Advances in

Operations Research

mansans

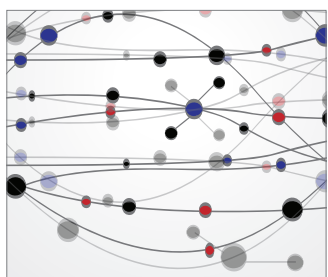

The Scientific World Journal
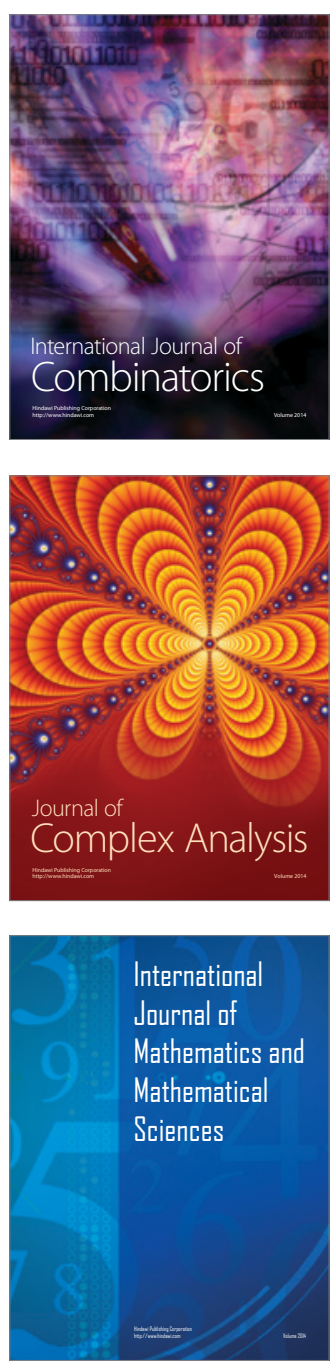
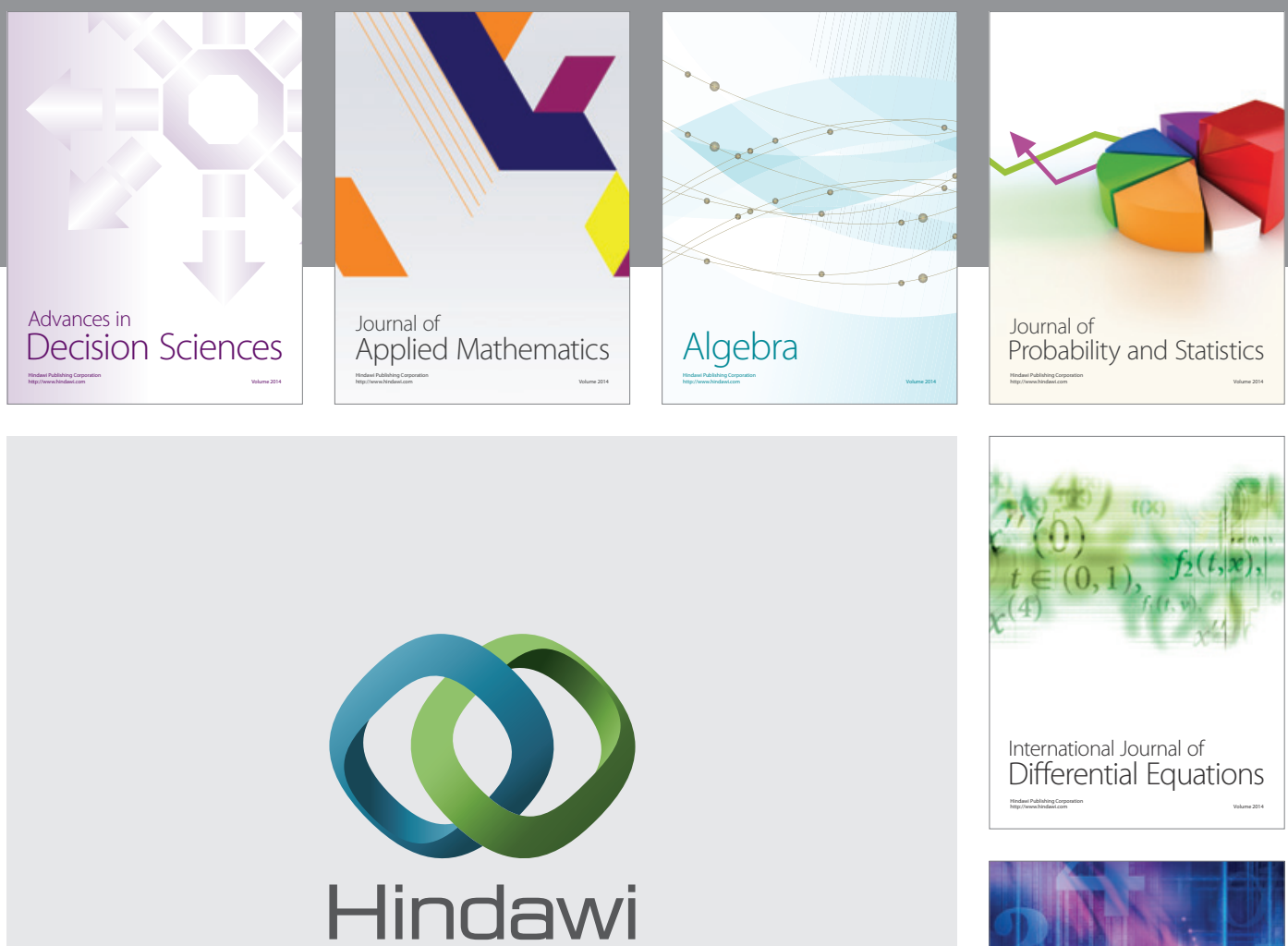

Submit your manuscripts at http://www.hindawi.com
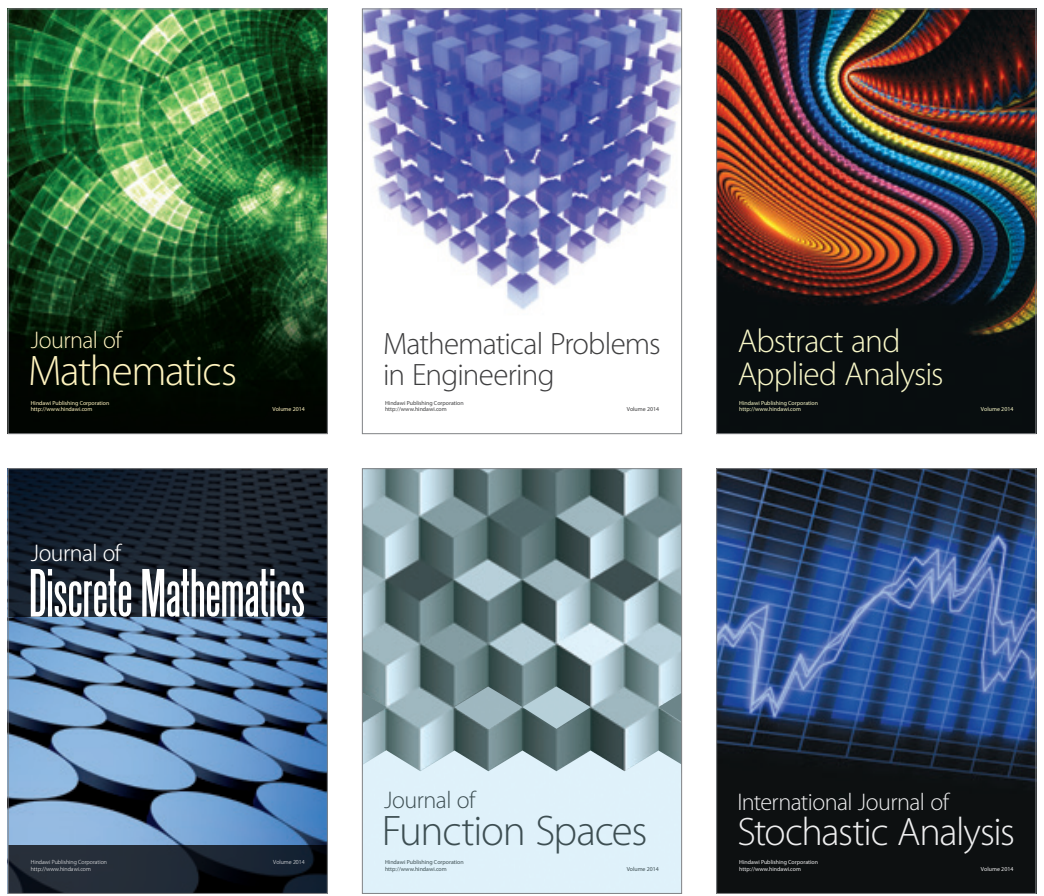

Journal of

Function Spaces

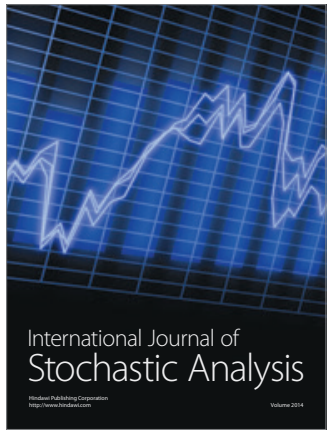

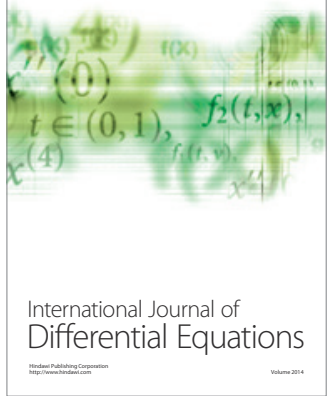
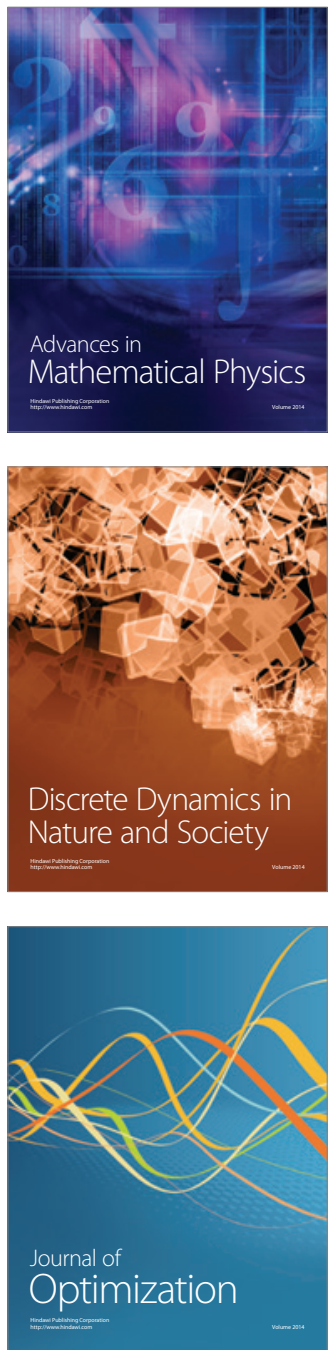